\title{
Estratégias utilizadas na assistência de enfermagem ao paciente adulto com doença crônica não transmissível: Uma revisão integrativa
}

\author{
Strategies usen in nursing care for adult patients with chronic non-communicable diseases: An \\ integrative review
}

Estrategias utilizadas en la atención de enfermería a pacientes adultos con enfermedades crónicas no transmisibles: Una revisión integradora

\section{Resumo}

O presente estudo teve como objetivo verificar as principais estratégias utilizadas na assistência de enfermagem ao adulto com doença crônica não transmissível na atenção primária a saúde. Trata-se de uma revisão integrativa da literatura, desenvolvida em janeiro de 2021. Foi utilizada a estratégia PVO, descritores controlados, cruzados com o operador booleano AND, nas bases: MEDLINE, BDENF, SCOPUS e CINAHL. Os artigos foram submetidos a um processo de filtragem constituído pelos critérios de inclusão: artigos completos e originais disponíveis na íntegra. Foram excluídos os estudos de revisão, duplicados, artigos que não respondam à pergunta de revisão, editoriais e literaturas cinzentas (publicações não-convencionais, evasivas). O gerenciamento das referências bibliográficas na seleção dos estudos primários incluídos na revisão se deu de forma manual. Foram identificados 1676 estudos e incluídos 15 artigos para compor a amostra dessa revisão. As estratégias encontradas foram organizadas em duas categorias temáticas: A primeira faz referência às consultas de enfermagem, visita domiciliar e uso de metodologias ativas durante o atendimento e estratégias desenvolvidas por meio de dispositivos móveis como o teleatendimento domiciliar, ambas fortificam o cuidado em saúde e ampliam a atenção a adultos com doenças crônicas. O papel do enfermeiro na atenção primária a saúde no que tange a consulta de enfermagem é essencial para promover um cuidado integral, equânime, resolutivo, humanizado, com foco no indivíduo e na família, assim como as atividades educativas, de gerenciamento da saúde e a utilização de aplicativos móveis são cruciais para promover e fortalecer o cuidado em saúde.

Palavras-chave: Atenção primária a saúde; Cuidados de enfermagem; Doença crônica.

\footnotetext{
Abstract

This study aimed to verify the main strategies used in nursing care for adults with non-communicable chronic disease in primary health care. This is an integrative literature review, developed in January 2021. The PVO strategy was used, with controlled descriptors, crossed with the Boolean operator AND, in the bases: MEDLINE, BDENF,
} 
SCOPUS and CINAHL. The articles were submitted to a filtering process consisting of the inclusion criteria: complete and original articles available in full. Review studies, duplicates, articles that do not answer the review question, editorials and gray literature (non-conventional, evasive publications) were excluded. The management of bibliographic references in the selection of primary studies included in the review was done manually. 1676 studies were identified and 15 articles were included to compose the sample of this review. The strategies found were organized into two thematic categories: The first refers to nursing consultations, home visits and the use of active methodologies during care and strategies developed through mobile devices such as home telecare, both of which strengthen health care and expand care for adults with chronic diseases. The role of nurses in primary health care regarding the nursing consultation is essential to promote comprehensive, equitable, resolute, humanized care, with a focus on the individual and the family, as well as educational activities, health management and Use of mobile apps is crucial to promoting and strengthening health care.

Keywords: Primary health care; Nursing care; Chronic disease.

\section{Resumen}

Este estudio tuvo como objetivo verificar las principales estrategias utilizadas en la atención de enfermería a adultos con enfermedades crónicas no transmisibles en la atención primaria de salud. Se trata de una revisión integradora de la literatura, desarrollada en enero de 2021. Se utilizó la estrategia PVO, con descriptores controlados, cruzados con el operador booleano AND, en las bases: MEDLINE, BDENF, SCOPUS y CINAHL. Los artículos fueron sometidos a un proceso de filtrado consistente en los criterios de inclusión: artículos completos y originales disponibles en su totalidad. Se excluyeron estudios de revisión, duplicados, artículos que no responden a la pregunta de revisión, editoriales y literatura gris (publicaciones evasivas no convencionales). La gestión de las referencias bibliográficas en la selección de los estudios primarios incluidos en la revisión se realizó de forma manual. Se identificaron 1676 estudios y se incluyeron 15 artículos para componer la muestra de esta revisión. Las estrategias encontradas se organizaron en dos categorías temáticas: la primera se refiere a las consultas de enfermería, visitas domiciliarias y el uso de metodologías activas durante la atención y estrategias desarrolladas a través de dispositivos móviles como la teleasistencia domiciliaria, las cuales fortalecen la atención de salud y amplían la atención a adultos con enfermedades crónicas. El papel del enfermero en la atención primaria de salud en relación con la consulta de enfermería es fundamental para promover una atención integral, equitativa, resolutiva, humanizada, con enfoque en el individuo y la familia, así como las actividades educativas, la gestión de la salud y el uso de aplicaciones móviles es fundamental para promover y fortalecer la atención de la salud.

Palabras clave: Atención primaria de salud; Atención de enfermería; Enfermedad crónica.

\section{Introdução}

No cenário de saúde, as doenças crônicas não transmissíveis (DCNT) têm elevada prevalência, sendo responsáveis por altas taxas de morbimortalidade em todo o mundo. Englobam doenças do aparelho circulatório, diabetes, câncer e doença respiratória crônica. Entre os principais fatores de risco para seu acometimento, destacam-se a inatividade física, o tabagismo, fatores genéticos, o consumo excessivo de álcool, a alimentação inadequada, entre outros, além de impactos econômicos para as famílias, comunidades e a sociedade em geral (Malta et al., 2019).

Segundo o Ministério da Saúde do Brasil, as DCNT são consideradas as principais causas de morbimortalidade em todo o mundo. Até o ano de 2020, à mortalidade por estes agravos corresponderão cerca de $73 \%$ das causas dos óbitos mundiais (Brasil, 2014). Em seu percurso as DCNTs, possuem uma série de especificidades, dentre os quais, fatores associados à melhora ou piora das manifestações clínicas; presença de múltiplas comorbidades associadas à doença principal; curso natural da doença longo e irregular; agudizações com necessidade de internações prolongadas e demandas que necessitam de uma abordagem multidimensional e multiprofissional até o fim da vida (Gallani, 2015).

A Estratégia de Saúde da Família (ESF) é o ponto chave da Atenção Primária à Saúde (APS), especialmente por reorganizar o modelo assistencial, com um alto grau de descentralização e capilaridade, por meio da atuação de uma equipe multiprofissional. Direcionada a resolutividade e longitudinalidade do cuidado, garante atendimento planejado com foco nas reais necessidades da comunidade a partir da territorialização (Brasil, 2017; Becker et al., 2018).

Em consonância a APS, a nova Política Nacional de Atenção Básica (PNAB), aprovada pela portaria n 2.436/2017, confere a composição mínima da equipe de saúde da família (eSF), formada preferencialmente por um médico e enfermeiro com especialidade em saúde da família e comunidade, um auxiliar ou técnico de enfermagem, agentes comunitários de saúde 
(ACS), podendo ser acrescentado a essa composição o agente comunitário de endemias (ACE) e os profissionais de saúde bucal. Em relação ao Núcleo Ampliado de Saúde da Família e Atenção Básica (NASF-AB), ele atua de forma complementar a equipe, fornecendo suporte clínico, sanitário e pedagógico. Dentro dessa equipe, o enfermeiro tem se revelado essencial para a expansão e consolidação da ESF (Brasil, 2017; Becker et al., 2018).

A atuação do enfermeiro na APS é um importante instrumento de mudança na prática do cuidado em doenças crônicas, com vistas a um atendimento pautado na humanização, considera o indivíduo em sua singularidade e promove a sua autonomia no processo saúde-doença. As ações do enfermeiro dentro da APS estão pautadas em fornecer um cuidado integral ao indivíduo e família em todos os ciclos da vida, realizar consultas de enfermagem garantindo uma assistência resoluta e de qualidade, além de contribuir com os demais membros da equipe (Ferreira et al., 2018).

As ações de promoção à saúde que são essenciais entre as atribuições do enfermeiro, fornecem um olhar para melhoria da qualidade de vida da população. As ações devem considerar os determinantes sociais de saúde e como esses impactam na vida da comunidade, respeitando a autonomia e a singularidade dos sujeitos. Desse modo, emergem a necessidade desses profissionais da saúde que compreendam a indispensabilidade da continuidade de cuidados e que isso não se estabeleça somente na prática assistencial como usualmente, mas também na coordenação do cuidado (Brasil, 2018; Becker et al., 2018).

Neste sentido, se faz importante a análise de estudos que abordem o papel do enfermeiro no cuidado a adultos em condições crônicas de saúde, principalmente no que tange a utilização de estratégias na APS e como podem ser replicadas no cuidado em enfermagem. Assim, esta pesquisa tem como objetivo verificar as principais estratégias utilizadas na assistência de enfermagem ao adulto com DCNT na Atenção Primária à Saúde.

\section{Metodologia}

O método de síntese do conhecimento adotado foi uma revisão integrativa da literatura, que permite a formulação de conclusões gerais a respeito de determinada área do conhecimento, mediante uma síntese de estudos publicados na perspectiva em estudo (Souza; Silva; Carvalho, 2010).

O estudo compreendeu as seguintes etapas: I) identificação do tema e seleção da hipótese ou questão de pesquisa para a elaboração da revisão integrativa; II) estabelecimento de critérios para inclusão e exclusão de estudos/amostragem ou busca na literatura; III) definição das informações a serem extraídas dos estudos selecionados/categorização dos estudos; IV) avaliação dos estudos incluídos na revisão integrativa; V) interpretação dos resultados; VI) apresentação da revisão/síntese do conhecimento (Mendes; Silveira; Galvão, 2008).

$\mathrm{Na}$ fase um, para a seguinte revisão integrativa foi elaborada a questão de pesquisa: Quais estratégias são utilizadas na assistência de enfermagem ao adulto com DCNT na atenção primária a saúde? Em busca de um resultado claro e que abordasse os aspectos do estudo, utilizou-se a estratégia Population, Variables and Outcomes (PVO) para busca dos artigos. 
Quadro 1 - Descritores de assunto localizados no DeCS e MeSH para os componentes da pergunta de pesquisa segundo a estratégia PVO. Crato, CE, Brasil, 2021.

\begin{tabular}{|c|c|c|c|}
\hline Sigla & Conteúdo & $\begin{array}{l}\text { DeCS Termos/ } \\
\text { MEDLINE via Pubmed, CINAHL } \\
\text { e BDENF }\end{array}$ & $\begin{array}{l}\text { MeSH termos/ } \\
\text { SCOPUS }\end{array}$ \\
\hline \multirow[t]{2}{*}{$\mathbf{P}$} & $\begin{array}{lr}\text { Pacientes } & \text { com } \\
\text { doenças crônicas } \\
\text { não transmissíveis } \\
(\mathrm{DCNT})\end{array}$ & Doença Crônica & Chronic Disease \\
\hline & & AND & AND \\
\hline \multirow[t]{2}{*}{$\mathbf{V}$} & $\begin{array}{l}\text { Atenção primária a } \\
\text { saúde }\end{array}$ & Atenção Primária a Saúde & Primary Health Care \\
\hline & & AND & AND \\
\hline $\mathbf{O}$ & $\begin{array}{ll}\text { Assistência } & \text { de } \\
\text { Enfermagem } & \end{array}$ & Cuidados de Enfermagem & Nursing Care \\
\hline \multicolumn{2}{|r|}{$\begin{array}{r}\text { Total de estudos por } \\
\text { base: }\end{array}$} & $\begin{array}{l}\text { BDENF (61 estudos); } \\
\text { MEDLINE (321 estudos); } \\
\text { CINAHL (661 estudos) }\end{array}$ & SCOPUS (633 estudos) \\
\hline
\end{tabular}

Fonte: Autores (2021).

As bases selecionadas no estudo se deram através da sua relevância na saúde e na enfermagem, sendo: Medical Literature Analysis and Retrieval System Online (MEDLINE) via Pubmed, Banco de Dados em Enfermagem (BDENF), National Library of Medicine National, SciVerse (SCOPUS), Cumulative Index to Nursing and Allied Health Literature (CINAHL). A busca foi realizada por meio do Portal de Periódicos da CAPES e a Biblioteca Virtual da Saúde (BVS).

Na fase dois, foram utilizados os Descritores em Ciências da Saúde (DeCs) e os Medical Subject Headings (MeSH) na busca dos artigos nas bases. Foram utilizados no DeCs: doença crônica, atenção primária a saúde e cuidados de enfermagem. Em relação ao MeSH: Chronic Disease, Primary Health Care e Nursing Care com o operador booleano AND. A busca aconteceu de forma pareada, com a presença de uma terceira pessoa para verificação dos dados, em janeiro de 2021, em um mestrado acadêmico de enfermagem no Nordeste do Brasil.

Os artigos foram submetidos a um processo de filtragem constituído pelos critérios de inclusão: artigos completos e originais disponíveis na íntegra. Foram excluídos os estudos de revisão, duplicados, artigos que não respondam à pergunta de revisão, editoriais e literaturas cinzentas (publicações não-convencionais, evasivas). Não foi preestabelecida uma delimitação temporal e de idiomas a fim de encontrar o maior número de artigos relacionados ao tema.

Para as bases MEDLINE, CINAHL e BDENF foram utilizadas as estratégias de busca: "Doença Crônica" AND “Atenção Primária a Saúde" AND "Cuidados de Enfermagem". Na BDENF foram identificados 61 achados. Na MEDLINE foram identificados 321 estudos e na CINAHL, foram identificados 661 estudos. Para a base SCOPUS foram utilizadas as estratégias de busca: "Chronic Disease" AND "Primary Health Care" AND "nursing care", sendo identificados 633 estudos.

Em suma, foram identificados 1.676 registros, destes 669 estudos foram removidos por duplicação, após a leitura exaustiva de título e resumo, foram excluídos 937 artigos, restando 70 estudos que contemplaram os critérios de elegibilidade, destes nove eram repetidos, 18 estudos incompletos, 7 estudos de outra natureza e 21 fugiam do tema proposto, sendo incluídos 15 estudos. 
Foi utilizado o fluxograma de Preferred Reporting Itens for Systematic Review and Meta-Analyses (PRISMA) para a descrição das etapas de seleção de busca e seleção dos estudos, como pode ser visto na Figura 1.

Figura1 - Fluxograma de seleção dos estudos, adaptado segundo Preferred Reporting Itens for Systematic Review and MetaAnalyses (PRISMA). Crato, CE, Brasil, 2021.

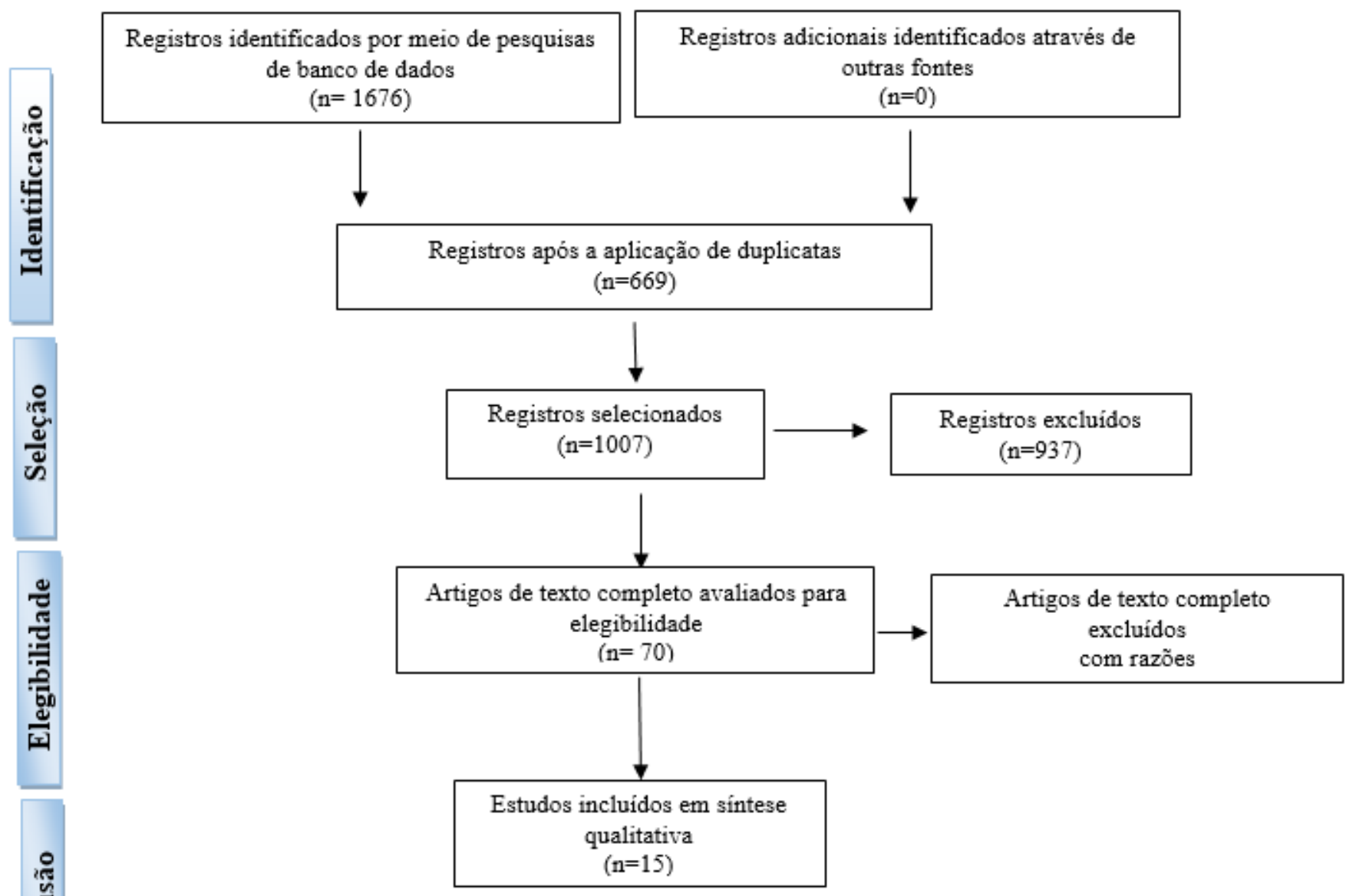

Fonte: Autores (2021).

O gerenciamento das referências bibliográficas na seleção dos estudos primários incluídos na revisão se deu de forma manual. Na fase três, os estudos primários incluídos na revisão tiveram as informações extraídas quanto ao autor, ano, local de publicação, idioma, periódico, nível de evidência, título do estudo e estratégia utilizada. Ademais, os dados foram categorizados em temáticas, classificadas por semelhança de conteúdo.

Os estudos foram categorizados conforme os sete níveis de evidência: nível 1, revisão sistemática ou metanálise de ensaios clínicos randomizados controlados / diretrizes clínicas que contenham os estudos de revisão supracitados; nível 2, ensaio clínico randomizado controlado e bem delineado; nível 3, ensaio clínico controle, mas sem randomização; nível 4, estudos de caso-controle ou coorte bem delineados; nível 5, revisão sistemática de estudos descritivos e qualitativos; nível 6, estudos descritivos ou qualitativos; e nível 7 opinião de especialistas (Melnyk et al., 2014). 


\section{Resultados}

Compuseram a amostra final 15 artigos que abordaram estratégias utilizadas na atenção primária a saúde para a assistência de enfermagem ao adulto com DCNT. Os estudos evidenciaram condições como: hipertensão arterial sistêmica, diabetes mellitus, câncer e pacientes com risco cardiovascular.

Seis foram publicados no Brasil e nove no exterior. Foi realizada a categorização dos estudos primários quanto ao delineamento autor/ano, país, idioma/periódico, nível de evidência, título do estudo e estratégia utilizada.

Os estudos objetivaram descrever a utilização de estratégias para o cuidado de enfermagem ao adulto com DCNT na APS. Foram publicados no período entre 2007 a 2020, correspondendo a 01 estudo publicado em 2007 (7\%), 02 publicados em 2012 (13\%), 02 em 2017 (13\%), 05 em 2018 (34\%), 03 em 2019 (20\%) e 02 estudos em 2020 (13\%). Em relação ao desenho metodológico abrangeram estudos descritivos e exploratórios, pesquisas mistas, estudos de intervenção, estudos-piloto e ensaios clínicos controlados randomizados. Quanto ao nível de evidência, 08 estudos corresponderam predominantemente ao nível VI (53\%), 05 estudos ao nível II (34\%) e 02 estudos nível III (13\%).

Quadro 2 - Categorização dos estudos primários incluídos na revisão. Crato, CE, Brasil, 2021. (N=15).

\begin{tabular}{|c|c|c|c|c|c|c|c|}
\hline Estudo & $\begin{array}{l}\text { Autor/ } \\
\text { Ano }\end{array}$ & Local & Idioma/Periódico & $\begin{array}{l}\text { Nível de } \\
\text { evidência }\end{array}$ & Título do estudo & $\begin{array}{l}\text { Estratégia } \\
\text { utilizada }\end{array}$ & $\begin{array}{l}\text { Desenho } \\
\text { Metodológico }\end{array}$ \\
\hline E1 & $\begin{array}{l}\text { Sikorsk } \\
\text { ii, et } \\
\text { al., } \\
2007 .\end{array}$ & $\begin{array}{l}\text { Indiana } \\
\text { EUA }\end{array}$ & $\begin{array}{l}\text { Inglês/ Journal of } \\
\text { Pain and } \\
\text { Symptom } \\
\text { Management }\end{array}$ & II & $\begin{array}{l}\text { Symptom } \\
\text { Management for } \\
\text { Cancer Patients: } \\
\text { A Trial } \\
\text { Comparing Two } \\
\text { Multimodal } \\
\text { Interventions }\end{array}$ & $\begin{array}{l}\text { Gerenciamento } \\
\text { de sintomas } \\
\text { assistido por } \\
\text { enfermagem } \\
\text { (NASM) e } \\
\text { gerenciamento } \\
\text { automatizado } \\
\text { de sintomas } \\
\text { por telefone } \\
\text { (ATSM) }\end{array}$ & $\begin{array}{l}\text { Ensaio Clínico } \\
\text { randomizado }\end{array}$ \\
\hline E2 & $\begin{array}{l}\text { Peeters, } \\
\text { et al., } \\
2012 .\end{array}$ & Holanda & $\begin{array}{l}\text { Inglês/ Journal of } \\
\text { Clinical Nursing }\end{array}$ & VI & $\begin{array}{l}\text { Factors } \\
\text { influencing the } \\
\text { adoption of home } \\
\text { telecare by elderly } \\
\text { or } \\
\text { chronically ill } \\
\text { people: a national } \\
\text { survey }\end{array}$ & $\begin{array}{l}\text { Tele- } \\
\text { atendimento } \\
\text { domiciliar }\end{array}$ & $\begin{array}{l}\text { Estudo } \\
\text { descritivo }\end{array}$ \\
\hline E3 & $\begin{array}{l}\text { Colósi } \\
\text { mo, et } \\
\text { al., } \\
2012 .\end{array}$ & $\begin{array}{l}\text { São } \\
\text { Paulo/Bras } \\
\text { il }\end{array}$ & $\begin{array}{l}\text { Português/Rev } \\
\text { Escola de } \\
\text { Enfermagem- } \\
\text { USP }\end{array}$ & II & $\begin{array}{l}\text { Atuação da } \\
\text { enfermeira eleva o } \\
\text { controle } \\
\text { de hipertensos e } \\
\text { diminui o efeito } \\
\text { do avental branco }\end{array}$ & $\begin{array}{l}\text { Medida } \\
\text { residencial da } \\
\text { pressão arterial } \\
\text { (MRPA) }\end{array}$ & $\begin{array}{l}\text { Estudo } \\
\text { comparativo } \\
\text { randomizado }\end{array}$ \\
\hline E4 & $\begin{array}{l}\text { Vest, et } \\
\text { al., } \\
2017 .\end{array}$ & $\begin{array}{l}\text { Western/ } \\
\text { New Yor }\end{array}$ & $\begin{array}{lr}\text { Inglês/Primary } \\
\text { Health Care } \\
\text { Research \& } \\
\text { Development }\end{array}$ & VI & $\begin{array}{l}\text { Nurse } \\
\text { perspectives on } \\
\text { the } \\
\text { implementation of } \\
\text { routine } \\
\text { telemonitoring for } \\
\text { high-risk diabetes } \\
\text { patients in a }\end{array}$ & $\begin{array}{l}\text { Telemonitoram } \\
\text { ento de rotina }\end{array}$ & $\begin{array}{l}\text { Estudo } \\
\text { qualitativo }\end{array}$ \\
\hline
\end{tabular}




\begin{tabular}{|c|c|c|c|c|c|c|c|}
\hline & & & & & $\begin{array}{l}\text { primary } \\
\text { setting }\end{array}$ & & \\
\hline E5 & $\begin{array}{l}\text { Alencar } \\
\text {, et al., } \\
2017 .\end{array}$ & $\begin{array}{l}\text { Piauí/ } \\
\text { Brasil }\end{array}$ & $\begin{array}{l}\text { Inglês/ Journal of } \\
\text { Nursing }\end{array}$ & VI & $\begin{array}{l}\text { Nursing } \\
\text { consultation in the } \\
\text { perspective of } \\
\text { users with } \\
\text { diabetes mellitus } \\
\text { in the family } \\
\text { health strategy }\end{array}$ & $\begin{array}{l}\text { Consulta de } \\
\text { Enfermagem }\end{array}$ & $\begin{array}{l}\text { Estudo } \\
\text { descritivo e } \\
\text { exploratório }\end{array}$ \\
\hline E6 & $\begin{array}{l}\text { Ribeiro } \\
\text {, et al., } \\
2018 .\end{array}$ & $\begin{array}{l}\text { Ceilândia/ } \\
\text { Brasil }\end{array}$ & $\begin{array}{ll}\text { Português/ Rev da } \\
\text { Escola } & \text { de } \\
\text { Enfermagem } & \text { da } \\
\text { USP } & \end{array}$ & VI & $\begin{array}{lr}\text { Síndrome } & \text { do } \\
\text { idoso frágil } & \text { em } \\
\text { idosos } & \text { com } \\
\text { doenças } & \text { crônicas } \\
\text { na } & \text { Atenção } \\
\text { Primária } & \end{array}$ & $\begin{array}{l}\text { Diagnóstico de } \\
\text { Enfermagem }\end{array}$ & $\begin{array}{l}\text { Estudo } \\
\text { descritivo } \mathrm{e} \\
\text { quantitativo }\end{array}$ \\
\hline E7 & $\begin{array}{l}\text { Queiro, } \\
\text { et al., } \\
2018 .\end{array}$ & $\begin{array}{l}\text { Natal/Brasi } \\
1\end{array}$ & $\begin{array}{lr}\text { Português/ } & \text { Rev } \\
\text { Brasileira } & \text { de } \\
\text { Enfermagem } & \end{array}$ & VI & $\begin{array}{l}\text { Percepção de } \\
\text { trabalhadores de } \\
\text { enfermagem sobre } \\
\text { o cuidado ao idoso } \\
\text { portador de } \\
\text { hipertensão } \\
\text { arterial sistêmica }\end{array}$ & $\begin{array}{l}\text { Territorializaçã } \\
\text { o/ Vínculo } \\
\text { usuário } \\
\text { equipe }\end{array}$ & $\begin{array}{l}\text { Estudo } \\
\text { descritivo } \\
\text { qualitativo }\end{array}$ \\
\hline E8 & $\begin{array}{l}\text { Becker, } \\
\text { et al., } \\
2018 .\end{array}$ & $\begin{array}{l}\text { Santa } \\
\text { Catarina/B } \\
\text { rasil }\end{array}$ & $\begin{array}{lr}\text { Português/ } & \text { Rev } \\
\text { Brasileira } & \text { de } \\
\text { Enfermagem } & \end{array}$ & VI & $\begin{array}{lr}\text { Práticas } & \text { de } \\
\text { cuidados } & \text { dos } \\
\text { enfermeiros } & \text { a } \\
\text { pessoas } & \text { com } \\
\text { Doenças } & \text { Crônicas } \\
\text { Não } & \\
\text { Transmissíveis }\end{array}$ & $\begin{array}{l}\text { Visita } \\
\text { domiciliar/ } \\
\text { atendimento } \\
\text { individual } \\
\text { coletivo }\end{array}$ & $\begin{array}{l}\text { Estudo } \\
\text { qualitativo }\end{array}$ \\
\hline E9 & $\begin{array}{l}\text { Mallow } \\
\text {, et al., } \\
2018 .\end{array}$ & $\begin{array}{l}\text { Estados } \\
\text { Unidos }\end{array}$ & $\begin{array}{l}\text { Inglês/ } \\
\text { International } \\
\text { Journal of } \\
\text { Nursing Sciences }\end{array}$ & III & $\begin{array}{l}\text { The effectiveness } \\
\text { of mI SMART: A } \\
\text { nurse practitioner } \\
\text { led technology } \\
\text { intervention for } \\
\text { multiple chronic } \\
\text { conditions in } \\
\text { primary care }\end{array}$ & MI Smart & $\begin{array}{l}\text { Ensaio Clínico } \\
\text { Controle }\end{array}$ \\
\hline E10 & $\begin{array}{l}\text { Watson } \\
\text {, et al., } \\
2018 .\end{array}$ & Inglaterra & $\begin{array}{l}\text { Inglês/European } \\
\text { Journal of } \\
\text { Oncology Nursing }\end{array}$ & II & $\begin{array}{l}\text { Supporting } \\
\text { prostate cancer } \\
\text { survivors in } \\
\text { primary care: } \\
\text { Findings from a } \\
\text { pilot } \\
\text { trial of a nurse-led } \\
\text { psycho- } \\
\text { educational } \\
\text { intervention } \\
\text { (PROSPECTIV) }\end{array}$ & $\begin{array}{l}\text { Intervenção } \\
\text { psicoeducativa }\end{array}$ & $\begin{array}{l}\text { Ensaio clínico } \\
\text { randomizado } \\
\text { controlado }\end{array}$ \\
\hline E11 & $\begin{array}{l}\text { Silva, } \\
\text { et al., } \\
2019 .\end{array}$ & $\begin{array}{l}\text { Piauí/Brasi } \\
1\end{array}$ & $\begin{array}{l}\text { Português/ Rev de } \\
\text { Enfermagem } \\
\text { UFPE }\end{array}$ & VI & $\begin{array}{lr}\text { Intervenção } & \text { de } \\
\text { Saúde } & \text { sobre } \\
\text { Hipertensão } & \text { e } \\
\text { Diabetes } & \end{array}$ & $\begin{array}{l}\text { Metodologia } \\
\text { do Arco de } \\
\text { Charles } \\
\text { Maguerez }\end{array}$ & $\begin{array}{l}\text { Estudo } \\
\text { qualitativo, } \\
\text { descritivo }\end{array}$ \\
\hline
\end{tabular}




\begin{tabular}{|c|c|c|c|c|c|c|c|}
\hline E12 & $\begin{array}{l}\text { Angwe } \\
\text { nyi PH, } \\
\text { et al., } \\
2019 .\end{array}$ & $\begin{array}{l}\text { Malawi, } \\
\text { África } \\
\text { Oriental }\end{array}$ & $\begin{array}{l}\text { Inglês/ Journal of } \\
\text { Advanced } \\
\text { Nursing }\end{array}$ & VI & $\begin{array}{l}\text { Patient-provider } \\
\text { perspectives on } \\
\text { self management } \\
\text { support and } \\
\text { patient } \\
\text { empowerment in } \\
\text { chronic care: A } \\
\text { mixed-methods } \\
\text { study in a rural } \\
\text { sub-Saharan } \\
\text { setting }\end{array}$ & $\begin{array}{l}\text { Autogestão do } \\
\text { cuidado }\end{array}$ & $\begin{array}{l}\text { Pesquisa } \\
\text { descritiva } \\
\text { simultânea de } \\
\text { métodos } \\
\text { mistos. }\end{array}$ \\
\hline E13 & $\begin{array}{l}\text { Casaña } \\
\text { s, et al., } \\
2019 .\end{array}$ & $\begin{array}{l}\text { Barcelona, } \\
\text { Espanha }\end{array}$ & $\begin{array}{lr}\text { Inglês/ } & \text { BMC } \\
\text { Health } & \text { Services } \\
\text { Research } & \end{array}$ & II & $\begin{array}{l}\text { Effectiveness of a } \\
\text { psychoeducation } \\
\text { group intervention } \\
\text { conducted by } \\
\text { primary healthcare } \\
\text { nurses in patients } \\
\text { with depression } \\
\text { and physical } \\
\text { comorbidity: } \\
\text { study protocol for } \\
\text { a } \\
\text { randomizedcontro } \\
\text { lled trial }\end{array}$ & $\begin{array}{l}\text { Grupo } \\
\text { psicoeducacion } \\
\text { al }\end{array}$ & $\begin{array}{l}\text { Ensaio clínico } \\
\text { controlado e } \\
\text { randomizado }\end{array}$ \\
\hline E14 & $\begin{array}{l}\text { Westla } \\
\text { nd, et } \\
\text { al., } \\
2020 \text {. }\end{array}$ & Holanda & $\begin{array}{l}\text { Inglês/European } \\
\text { Journal of } \\
\text { Cardiovascular } \\
\text { Nursing }\end{array}$ & II & $\begin{array}{l}\text { Effectiveness of } \\
\text { the nurse-led } \\
\text { Activate } \\
\text { intervention in } \\
\text { patients at risk of } \\
\text { cardiovascular } \\
\text { disease in primary } \\
\text { care: a cluster- } \\
\text { randomised } \\
\text { controlled trial }\end{array}$ & $\begin{array}{l}\text { Intervenção } \\
\text { Activate }\end{array}$ & $\begin{array}{l}\text { Ensaio clínico } \\
\text { controlado e } \\
\text { randomizado }\end{array}$ \\
\hline E15 & $\begin{array}{l}\text { Zimans } \\
\text { ky, et } \\
\text { al., } \\
2020 .\end{array}$ & Alemanha & $\begin{array}{l}\text { Inglês/ Journal of } \\
\text { Family Nursing }\end{array}$ & III & $\begin{array}{l}\text { Effects of } \\
\text { implementing a } \\
\text { brief Family } \\
\text { nursing } \\
\text { intervention with } \\
\text { hospitalized } \\
\text { oncology patients } \\
\text { and their families } \\
\text { in germany: A } \\
\text { quasi- } \\
\text { experimental } \\
\text { study }\end{array}$ & $\begin{array}{l}\text { Enfermagem } \\
\text { Familiar }\end{array}$ & $\begin{array}{l}\text { Estudo quase } \\
\text { experimental }\end{array}$ \\
\hline
\end{tabular}

Fonte: Autores (2021).

Os dados foram organizados em duas categorias temáticas, classificadas por semelhança de conteúdo. A primeira categoria faz referência as consultas de enfermagem e a segunda trata de estratégias desenvolvidas por meio de dispositivos móveis. 
Os estudos abordaram sobre o uso de estratégias maximizadoras para adesão e controle de pacientes hipertensos, intervenção em pacientes hipertensos e diabéticos com base em parâmetros da metodologia do Arco de Charles Maguerez, práticas de cuidado na consulta de enfermagem a pacientes crônicos, importância de grupos de psicoeducação para reduzir sintomas depressivos em pacientes crônicos e apoio a sobreviventes de câncer de próstata na atenção primária.

\section{Categoria 2- Estratégias desenvolvidas por meio de dispositivos móveis para apoiar a autogestão do cuidado}

Os estudos discorreram sobre a utilização de estratégias para o cuidado em enfermagem com foco na autogestão do cuidado de pacientes crônicos, planejamento, acompanhamento da assistência, avaliação de intervenções multimodais de sintomas em relação ao tratamento convencional de pacientes com câncer, uso da intervenção activate para promoção do componente atividade física e teleatendimento domiciliar e de rotina por pacientes com condições crônicas.

\section{Discussão}

Os estudos que tinham como foco a consulta de enfermagem como instrumento de mudança para o cuidado a pessoas com doenças crônicas e a importância da educação continuada e de novas abordagens de cuidado nos diversos níveis de atenção à saúde reforçam o escopo da prática de enfermagem em APS tanto no atendimento individual quanto coletivo. Não obstante, a visita domiciliar, o atendimento individual e coletivo com foco nos determinantes sociais de saúde favorecem práticas de promoção e educação da saúde, acolhimento humanizado e conhecimento das condições de vida das pessoas.

Nesse sentido, um estudo foi desenvolvido com diabéticos e evidenciou a importância da consulta de enfermagem ao paciente diabético na atenção primária a saúde, pois reforça o conhecimento da comunidade sobre a doença e suas complicações e fortalece o diagnóstico e acompanhamento precoce. Por outro lado, um estudo desenvolvido no município de Natal, Rio Grande do Norte, Brasil, apontou que territorialização, parcerias, vínculo do usuário com a equipe, foco da assistência centrado na doença, formação acadêmica pautada no modelo biomédico, inexistência de intersetorialidade e descontinuidade do cuidado na rede assistencial, como elementos definidores da assistência de enfermagem na Estratégia Saúde da Família, convergentes com a necessidade de um cuidado integral (Alencar et al., 2017; Becker et al., 2018; Queiroz et al., 2019).

Nesse sentido, acerca da adesão e controle de pacientes hipertensos no município de São Paulo, Brasil, um estudo abordou estratégias potencializadoras como a medida casual e a medida residencial da pressão arterial (MRPA). Os participantes foram divididos em grupo intervenção e controle, respectivamente, com participação em atividades educativas e seguimento da rotina de atendimentos da unidade de saúde. Os pacientes que participaram das intervenções educativas apresentaram decréscimo significante na pressão arterial sistólica e diastólica, após as intervenções educativas de MRPA (Colósimo et al., 2012).

A metodologia do arco de Charles Maguerez foi considerada satisfatória e positiva com pacientes hipertensos e diabéticos em uma unidade de saúde do Estado do Piaú, Brasil, visto que propicia uma maior interação do profissional da APS com a população adscrita no território, pelo processo de territorialização, com a identificação das reais necessidades da comunidade (Silva et al., 2019).

No que tange a utilização de diagnósticos de enfermagem foi visto que o diagnóstico "Síndrome do Idoso Frágil" em idosos com diabetes mellitus e hipertensão arterial sistêmica, obteve como principais categorias definidoras: memória prejudicada, mobilidade física prejudicada, fadiga e déficit de autocuidado. Esses resultados fortificam a importância do diagnóstico e tratamento precoce de condições crônicas de saúde, a fim de prevenir as limitações e incapacidades e fortalecer medidas de autocuidado (Ribeiro et al., 2019). 
Um estudo realizado na Alemanha enfatiza o valor da enfermagem familiar com base nos modelos de Calgary, que abordam aspectos de melhoria da saúde e bem- estar familiar dentro do contexto hospitalar oncológico. Os efeitos da enfermagem da família são percebidos nos pacientes e familiares, nos aspectos de humor, ansiedade, queixas físicas e suporte social. A implementação aconteceu em um período de 06 meses e os profissionais da unidade oncológica passaram por um treinamento organizado pela equipe da enfermagem familiar com foco nos aspectos de atitude empática e comunicativa, uso de conversas terapêuticas nos momentos com os familiares e prevenção de erros de enfermagem. Os resultados indicaram que são necessários mais estudos em outros ambientes para garantir a eficácia da enfermagem familiar no contexto hospitalar (Zimansky et al., 2020).

Dois estudos abordaram a importância de ações psicoeducativas no cuidado de enfermagem (Casañas et al., 2019; Watson et al., 2018). Um artigo abordou aplicação de atividade psicoeducativas em pacientes crônicos com transtornos depressivos na atenção primária. A intervenção psicoeducativa foi baseada em um protocolo de 12 sessões semanais de 90 minutos conduzidas por duas enfermeiras da atenção básica. Os principais objetivos da sessão ofereceram informação sobre sintomas depressivos e condições crônicas, bem como sua associação, orientações sobre alimentação, sono e repouso, prática de atividade física, adesão terapêutica, técnicas de respiração, resolução de problemas e autoestima. As intervenções demonstraram ser eficazes na redução dos sintomas depressivos, e melhora da adesão terapêutica (Casañas et al., 2019).

Outro artigo tratou da intervenção psicoeducativa com base em uma abordagem de autogestão, sustentado pela teoria social Cognitiva de Bandura, liderada por uma enfermeira na atenção primária a pacientes que superaram o câncer de próstata. A intervenção abrangeu quatro domínios: compreender o contexto de tratamento do câncer de próstata, autogestão, ativação comportamental e restruturação cognitiva. Os participantes foram divididos em dois grupos: intervenção e controle. O grupo intervenção relatou melhora na autoestima, maior probabilidade de procurar um serviço de atenção primária para solucionar dúvidas e procurar atendimento (Watson et al., 2018).

As tecnologias de informação e comunicação (TIC) vêm sendo amplamente utilizadas nos contextos educacionais e de saúde. Em virtude de um crescimento acelerado, as TIC favorecem e fortificam as relações da equipe de enfermagem com a população, a partir de uma assistência com mais qualidade, por meio de aparelhos portáteis, assistentes digitais pessoais e dispositivos de monitoramento de pacientes (Nascimento et al., 2020).

A intervenção MI Smart que integra várias tecnologias, foi desenvolvida, implementada e avaliada por enfermeiras em uma clínica comunitária de atenção primária nos Estados Unidos. Os participantes receberam um tablet Nexus7 e dispositivos de automonitoramento Bluetooth e três meses de serviço de internet. Os participantes receberam orientações de como utilizar os dispositivos e dados de glicose sanguínea, a pressão arterial e o peso foram coletados usando os dispositivos Bluetooth. Vídeos educacionais relacionado ao cuidado foram fornecidas por meio da plataforma MI Smart. Os resultados demonstraram melhora significativa entre os valores de pressão arterial e glicose antes e pós intervenção e os pacientes estão dispostos a melhorar sua condição clínica com o uso de tecnologia (Mallow et al., 2018).

Um estudo comparativo entre gerenciamento de sintomas assistido por enfermagem (NASM) ou gerenciamento automatizado de sintomas por telefone (ATSM) para pacientes com câncer submetidos à quimioterapia retratou que ambos os grupos alcançaram uma redução significativa na gravidade dos sintomas em relação ao início do estudo, e não houve diferença entre os grupos na gravidade dos sintomas em 10 semanas. Os resultados sugerem que tanto NASM quanto ATSM alcançaram uma redução clinicamente significativa na gravidade dos sintomas. O NASM pode ser mais eficaz do que o ATSM na retenção de pacientes com câncer de pulmão (Sikorskii et al., 2007).

A intervenção Activate e o fornecimento de apoio de autogestão a pacientes crônicos em ambientes com recursos limitados consistiu em casos de autogerenciamento a saúde. Os enfermeiros fizeram momentos educativos com os participantes sobre a conscientização do perfil de risco de doenças cardiovasculares (DCV) e seu nível de atividade física. 
Durante o período de intervenção, os pacientes foram instruídos a automonitorar sua atividade física diariamente usando um monitor de atividade pessoal e manter um registro de atividades. Em conclusão, a intervenção Activate liderada por enfermeiras não aumentou o nível de atividade física em pacientes com risco de DCV na atenção primária e o fornecimento de autogestão para ser ideal necessita de reformas a nível interpessoal e organizacional, incluindo; colaboração mútua pacienteprovedor, diversificando o acesso a recursos de apoio de autogestão e reestruturando grupos de apoio ao paciente para atender a diversas condições crônicas (Westland et al., 2020; Angwenyi et al., 2019).

Em consonância os dispositivos de teleatendimento domiciliar (TD) são mais favoráveis quando o cliente já recebe cuidados pessoais e/ou de enfermagem de longo prazo, mora sozinho e quando há contatos diários fixos através do sistema de teleatendimento domiciliar. O contato/ vínculo entre paciente e profissional são imprescindíveis para o sucesso do teleatendimento, bem como a inserção do TD nos cuidados primários em saúde (Peeters et al., 2012; Vest et al., 2017).

As limitações deste estudo voltam-se a etapa de seleção de periódicos, que ocorreu em diversas bases de dados e com expressivo número de documentos recuperados e periódicos relevantes para a área, contudo outras bases possíveis não foram consultadas. Além da análise e abordagem adotada neste estudo, outras possibilidades de análise e abordagens dos cuidados de enfermagem nas DCNT na APS também podem ser factíveis, com o desenvolvimento de novos comportamentos em relação ao cuidado em saúde. Como ponte forte da presente revisão, esta preenche uma lacuna atual de conhecimento ao reunir literaturas e fornecer uma visão geral das estratégias de cuidados de enfermagem às pessoas com DCNT na APS.

\section{Conclusão}

Verificou-se na literatura que as estratégias utilizadas ao adulto com DCNT na APS são voltadas para consultas de enfermagem, visita domiciliar e uso de metodologias ativas durante o atendimento e estratégias desenvolvidas por meio de dispositivos móveis como o teleatendimento domiciliar.

O papel do enfermeiro na atenção primária a saúde no que tange a consulta de enfermagem é essencial para promover um cuidado integral, equânime, resolutivo, humanizado, com foco no indivíduo e na família e embasado nos princípios do sistema único de saúde, assim como as atividades educativas, de gerenciamento da saúde e a utilização de aplicativos móveis são cruciais para promover e fortalecer o cuidado de pacientes com doenças crônicas não transmissíveis.

Espera-se que os resultados levantados nesse artigo possam contribuir para o debate da categoria e entidades de classe sobre a ampliação do trabalho do enfermeiro da APS. Fortalecer o trabalho do enfermeiro da APS com atribuições definidas pode ser um caminho para obter maior satisfação profissional, bem como contribuindo com a cobertura e o acesso universal à APS.

\section{Referências}

Alencar, D. C., et al. (2017). Nursing consultation in the perspective of users with diabetes mellitus in the family health strategy. $J$ Nurs UFPE on line 11(10):3749-56. https://periodicos.ufpe.br/revistas/revistaenfermagem/article/view/14005

Angwenyi, V., et al. (2019). Patient-provider perspectives on self management support and patient empowerment in chronic care: A mixed methods study in a rural sub Saharan setting. J Adv Nurs 75(11):2980-2994. 10.1111/jan.14116.

Becker, R. M., et al. (2018). Práticas de cuidados dos enfermeiros a pessoas com Doenças Crônicas Não Transmissíveis. Rev Bras Enferm 71(6):2643-9. http://dx.doi.org/10.1590/0034-7167-2017-0799

Brasil. (2014). Estratégia para o cuidado da pessoa com doença crônica: Diabetes Mellitus [Internet]. Brasília, DF: Ministério da Saúde; http://189.28.128.100/dab/docs/portal dab/publicacoes/caderno_36.pdf

Brasil. (2017). Portaria n 2.436, de 21 de setembro de 2017.Aprova a Política Nacional de Atenção Básica, estabelecendo a revisão de diretrizes para a organização da Atenção Básica, no âmbito do Sistema Único de Saúde (SUS). Diário Oficial da União. Brasília, DF, 22 set.

Brasil. (2018). Política Nacional de Promoção da SaúdeBrasília: Ministério da Saúde. Secretaria de Vigilância em Saúde. Secretaria de Atenção à Saúde. Anexo I da Portaria de Consolidação n. ${ }^{\circ}$ 2, de 28 de setembro de 2017. https://bvsms.saude.gov.br/bvs/publicacoes/politica_nacional_promocao_saude.pdf 
Casañas, R., et al. (2019). Effectiveness of a psychoeducation group intervention conducted by primary healthcare nurses in patients with depression and physical comorbidity: study protocol for a randomized controlled trial. BMC Health Services Research https://doi.org/10.1186/s12913-019-4198-7

Colósimo, F. C., et al. (2012). Atuação da enfermeira eleva o controle de hipertensos e diminui o efeito do avental branco. Rev Esc Enferm USP [Internet] [acesso em 19 de janeiro 2021]; 46(Esp):10-5. https://www.scielo.br/pdf/reeusp/v46nspe/02.pdf

Ferreira, S. R. S., et al. (2017). A complexidade do trabalho do enfermeiro na Atenção Primária à Saúde. Rev Bras de Enferm REBEN [Internet] [acesso em 09 de Julho de 2021]; 71(Supl 1):704-9. https://www.scielo.br/j/reben/a/qTVY5r3JLdL8xcTHNf9ZhxF/?lang=pt\&format=pdf.

Gallani, M. C. B. J. (2015). O enfermeiro no contexto das doenças crônicas. Rev. Latino-Am. Enfermagem Editorial. [Intenet]; [acesso em 19 de janeiro 2021]; 23(1):1-2. https://www.scielo.br/pdf/rlae/v23n1/pt_0104-1169-rlae-23-01-00001.pdf

Mallow, J. A., et al. (2018). The effectiveness of mI SMART: A nurse practitioner led technology intervention for multiple chronic conditions in primary care. International Journal of Nursing Sciences 5(2):131-137. https://doi.org/10.1016/j.ijnss.2018.03.009

Malta, D. C., et al. (2019). Probabilidade de morte prematura por doenças crônicas não transmissíveis, Brasil e regiões, projeções para 2025. Revista Brasileira de Epidemiologia 22:E190030. http://dx.doi.org/10.1590/1980-549720190030

Melnyk, T., et al. (2014). A importância da prática da psicologia baseada em evidências: aspectos conceituais, níveis de evidência, mitos e resistências. Revista Costarricense de Psicología. 33(2):79-92.

Mendes, K. D. S., Silveira, R. C. C. P., Galvão, C. M. (2008). Revisão integrativa: método de pesquisa para a incorporação de evidências na saúde e na enfermagem. Texto \&amp; Contexto - Enfermagem, 17(4):758-64. https://www.scielo.br/scielo.php?pid=s 010407072008000400018\&amp;script=sci_abstract\&amp;tlng=pt.

Nascimento, B. R. S., et al. (2020). Information and communication technologies: an emerging concept in nurses practices in basic care. Brazilian Journal of Production Engineering 6(6):01-10. Disponível em: https://periodicos.ufes.br/bjpe/article/view/30493.

Peeters, J. M., et al. (2012). Fatores que influenciam a adoção de teleatendimento domiciliar por idosos ou pessoas com doenças crônicas: uma pesquisa nacional. Journal of Clinical Nursing 21, 3183-3193. 10.1111 / j.1365-2702.2012.04173.x

Queiroz, R. F., et al.(2019). Percepção de trabalhadores de enfermagem sobre o cuidado ao idoso portador de hipertensão arterial sistêmica. Rev Bras Enferm. 72(Suppl 2):3-13. http://dx.doi.org/10.1590/0034-7167-2016-0681

Ribeiro, I. A., et al. (2019). Síndrome do idoso frágil em idosos com doenças crônicas na Atenção Primária. Rev Esc Enferm USP. 53:e03449. http://dx.doi.org/10.1590/S1980-220X2018002603449

Sikorskii, A., et al. (2007). Gerenciamento de sintomas para pacientes com câncer: um ensaio comparando duas intervenções multimodais. Journal of Pain and Symptom Management 34(3):253-264. 10.1016 / j.jpainsymman.2006.11.018

Silva, F. H. M., et al. (2019). Intervenção de saúde sobre hipertensão e diabetes. Rev enferm UFPE on line 13:e240593. https://doi.org/10.5205/19818963.2019.240593

Souza, M. T., Silva, M. D., Carvalho R. (2010). Revisão integrativa: o que é e como fazer. Einstein 8(1):102-106. https://www.scielo.br/pdf/eins/v8n1/pt -1679-4508-eins-8-1-0102.pdf

Vest, B. M., et al. (2017). Nurse perspectives on the implementation of routine telemonitoring for high-risk diabetes patients in a primary care setting. Pesquisa e desenvolvimento de cuidados primários de saúde 18:3-13. 10.1017 / S1463423616000190.

Watson, E. K., et al. (2018). Supporting prostate cancer survivors in primary care: Findings from a pilot trial of a nurse-led psycho-educational intervention (PROSPECTIV). Eur J Oncol Nurs 32:73-81. 10.1016/j.ejon.2017.12.002.

Westland, H., et al. (2020). Effectiveness of the nurse-led Activate intervention in patients at risk of cardiovascular disease in primary care: a clusterrandomised controlled trial. Eur J Cardiovasc Nurs 19(8):721-731. 10.1177 / 1474515120919547

Zimansky, M., et al. (2020). Effects of implementing a brief Family nursing intervention with hospitalized oncology patients and their families in germany: A quasi-experimental study. Journal of Family Nursing 26(4):346-357. 10.1177/ 1074840720967022. 